\title{
Fate of the rectum and distal colon after subtotal colectomy for ulcerative colitis ${ }^{1}$
}

\author{
B. I. KORELITZ, W. P. DYCK, AND F. M. KLION \\ From the Division of Gastroenterology, Department of Medicine, \\ The Mt Sinai Hospital and the Mt Sinai School of Medicine, New York, NY
}

Once the gastroenterologist and surgeon have agreed that there is an indication for definitive surgery in the patient with ulcerative colitis, they usually intend that the entire colon and rectum be resected. The operation of choice, then, should be a total proctocolectomy with ileostomy. In practice, however, a lesser initial procedure has been favoured in most cases, so that the subtotal colectomy with ileostomy has been the most frequently performed operation for ulcerative colitis in the past two decades. In some cases the distal segment would be resected electively soon afterwards, but in other cases the clinical course following the initial operation served to alter the original plan. The purpose of the present study was to determine the clinical behaviour and the later management of this residual segment, and to consider how its fate should influence the choice of surgical procedures for ulcerative colitis.

\section{MATERIAL AND METHOD}

The records of those patients with ulcerative colitis who had been admitted to the Mt Sinai Hospital between 1952 and 1963 were reviewed to select cases treated by onestage ileostomy and subtotal colectomy in whom the rectal segment was left behind (its proximal end having been brought out in the left lower quadrant as a mucous fistula). The diagnosis of ulcerative colitis was confirmed in these cases by the clinical course, characteristic radiological features on barium enema $x$-ray examination, and pathological examination of the surgical specimen. Excluded from the study were cases in the following categories: (1) those patients with inflammatory disease of the colon whose hospital records could not be found; (2) those patients in whom there was radiological evidence of granulomatous as opposed to ulcerative colitis; (3) those patients treated by total proctocolectomy; (4) those patients treated by ileostomy and subtotal colectomy when the colonic dissection was terminated proximal to the midsigmoid colon; (5) those patients treated by ileocolostomy or ileoproctostomy, with resection; and (6) those patients who died in the immediate postoperative period following ileostomy and subtotal colectomy.

${ }^{1}$ Assisted in part by the Ileitis and Colitis Foundation Inc.
The hospital records were further reviewed to gather information about the later status of the distal segment and whether and when an abdominoperineal resection was performed and if so the apparent indication. In most cases the private records of the attending gastroenterologist or surgeon or both were reviewed in order to acquire the necessary data. In other cases it was necessary to trace the patient or his family and hence to contact physicians or surgeons or hospital record rooms locally or at great distances to determine the late follow-up.

\section{RESULTS}

There were 136 patients who fulfilled the criteria for inclusion in this study. In $126(93.6 \%)$ the distal segment was eventually resected. The time intervals between the subtotal colectomy and the abdominoperineal resection were determined in 123 cases and are tabulated in Table I. The indications for re-

\section{T A B L E I}

INTERVAL BETWEEN SUBTOTAL COLECTOMY AND ARDOMINOPERINEAL RESECTION

\begin{tabular}{lc} 
Time & No. of Cases \\
\hline Immediate postoperatively & 5 \\
$2-6 \mathrm{mth}$ & 17 \\
$7 \mathrm{mth}-1 \mathrm{yr}$ & 26 \\
$1-2 \mathrm{yr}$ & 34 \\
$2-3 \mathrm{yr}$ & 17 \\
$4 \mathrm{yr}$ & 7 \\
$5 \mathrm{yr}$ & 5 \\
$7 \mathrm{yr}$ & 6 \\
$8-11 \mathrm{yr}$ & 5 \\
$16 \mathrm{yr}$ & 1 \\
Total & 123
\end{tabular}

moving the rectal segment are identified in Table II. When more than one indication existed, that which seemed more urgent was considered the primary while the others are listed as supporting.

In 112 of the 126 resected specimens, reports of pathological examinations were available for review. All revealed evidence of chronic ulcerative colitis, while in $\mathbf{4 2}$ there were findings of acute disease as 
T A B L E II

INDICATIONS FOR ABDOMINOPERINEAL RESECTION AFTER PREVIOUS ILEOSTOMY AND SUBTOTAL COLECTOMY FOR ULCERATIVE COLITIS

\begin{tabular}{|c|c|c|c|}
\hline \multirow[b]{2}{*}{ Indication for Resection } & \multicolumn{3}{|c|}{ No. of Cases } \\
\hline & Primary & Supporting & Total \\
\hline Bloody or purulent rectal discharge & 27 & 21 & 48 \\
\hline Rectal stricture & 33 & 8 & 41 \\
\hline Profuse rectal bleeding & 17 & - & 17 \\
\hline Systemic complications of residual ulcerative colitis & 9 & 3 & 12 \\
\hline Ileostomy complications & $3^{1}$ & 12 & 15 \\
\hline Perirectal complications & 6 & - & 6 \\
\hline Rectal polypi & 3 & 4 & 7 \\
\hline Recto-vaginal fistula & - & 5 & 5 \\
\hline Elective (active disease by sigmoidoscopy) & 27 & - & 27 \\
\hline Total & 125 & 53 & \\
\hline
\end{tabular}

${ }^{1}$ Abdominoperineal resection performed because of small bowel obstruction.

T A B L E III

CORRELATION BETWEEN INDICATIONS FOR SURGERY AND PATHOLOGICAL FINDINGS IN RECTAL SEGMENT AFTER ABDOMINOPERINEAL RESECTION

No. of Cases

\begin{tabular}{|c|c|c|c|c|}
\hline \multirow[b]{2}{*}{ Indication for Surgery } & \\
\hline & Total & Healing & Acute & Pseudopolypi \\
\hline Rectal stricture & 41 & 10 & 14 & 5 \\
\hline Bloody or purulent discharge & 48 & 14 & 15 & 6 \\
\hline Profuse rectal bleeding & 17 & 5 & 5 & 1 \\
\hline Elective & 27 & 5 & 6 & 5 \\
\hline Systemic complications & 12 & 3 & 6 & 2 \\
\hline Ileostomy complications & 15 & 2 & 4 & 2 \\
\hline Perirectal complications & 6 & 1 & 2 & 2 \\
\hline Recto-vaginal fistula & 5 & 0 & 1 & 0 \\
\hline
\end{tabular}

well. In 35 there was evident healing of the pathological process. Pseudopolypi were noted in 21.

There was no significant correlation between the symptoms or complications leading to the abdominoperineal resection and the severity of inflammation of the rectal mucosa as examined in the gross surgical specimen (Table III). The incidence of acute mucosal changes and of healing was similar whether the indication for surgery was bloody discharge, rectal haemorrhage, rectal stricture, or if the surgery was performed electively. Only when systemic complications of ulcerative colitis occurred were acute mucosal changes more likely to be found.

Of 35 cases where there was evidence of healing in the surgical specimen, the surgery had been performed electively without specific indication in eight and in another six for stricture, while in the remainder there had been clinical evidence of active disease.

In seven cases $(5.2 \%)$ the rectal segment was retained at the time of last follow-up, and no further surgery had been performed. One had had persistent ileostomy dysfunction and died two years after primary surgery of a pulmonary embolism. Of the other six, all had evidence of active ulcerative colitis in the rectal segment at the time of last examination. One of these had a rectal stricture, one had an ischiorectal abscess, and in the other four abdominoperineal resection had already been recommended.

In the three remaining cases $(2 \cdot 2 \%)$ the rectal segment was utilized for a reanastomosis, three, four, and 10 years after the initial operation. The first patient has had extension of disease to the previously normal appearing rectum with recurrent symptoms; the second developed a perirectal abscess after the reanastomosis but was only slightly inconvenienced one year thereafter; the third has remained well for eight years.

\section{COMMENT}

Once it has been concluded that surgical intervention, whether urgent or elective, is indicated in the management of ulcerative colitis, a new dilemma arises in considering the choice of operative procedures. Following partial surgical treatment by ileostomy and subtotal colectomy, the disease remains active in the rectal segment in $98 \%$. Bloody discharge persists in at least $52 \%$ and in one quarter 
of these the bleeding is profuse. In $33 \%$ the development of a rectal stricture causes concern that a carcinoma has developed; in another $5 \%$ polypsis causes similar concern. In $10 \%$ persistent or recurrent systemic complications of ulcerative colitis are attributable to active disease in the rectal segment, and in another $9 \%$ a perirectal suppurative complication or a rectovaginal fistula does not heal despite the previous resection and diversion of intestinal flow. In $12 \%$ there is recurrent ileostomy dysfunction, in one fifth of these frank small bowel obstruction; in some instances the obstruction is explained by adhesion between the small bowel and the residual distal segment. A small number, $4 \%$, require emergency resection of the distal segment immediately after the subtotal colectomy, usually because of massive haemorrhage but occasionally when this segment is considered the cause of small bowel obstruction. Ultimately, at least $94 \%$ require abdominoperineal resection. Other studies approached in a similar manner have shown almost as poor results (Mayo, Fly, and Connelly, 1956; Moss and Keddie, 1965).

This major surgical procedure carried a mortality risk of $3 \%$ (four cases). In two cases, death was attributable to a pulmonary embolism. In the other two cases death followed an emergency resection of the rectal segment soon after the subtotal colectomy because of continued haemorrhage; one of these patients also had cirrhosis and died in hepatic coma, while in the other, the indication for primary surgery had been toxic dilatation with perforation, and the patient's general condition remained poor.

The possibility of a complicating carcinoma of the rectum persists as long as the rectum is retained. In one 16-year-old girl from this study a carcinoma was unexpectedly found in the rectal segment two years after the subtotal colectomy and five years after the onset of ulcerative colitis. This is the only instance of carcinoma developing in the distal segment in this group of patients.

The advantages in performing a total proctocolectomy are evident. The patient is for all practical purposes cured of his ulcerative colitis by the one operation. Resection of the rectal segment removes the last site of active disease with its associated physical and emotional discomfort, its risk of carcinoma, the possibility of continued systemic complications, the increased likelihood of ileostomy dysfunction, and even small bowel obstruction due to adhesions, and the probability of requiring major surgery with renewed morbidity and mortality.

Despite the remote possibility of healing of ulcerative colitis in the residual segment following ileostomy and subtotal colectomy, there are some circumstances when this lesser procedure is pre- ferable. Even though the overall operative mortality rate is stated to be no greater for total proctocolectomy than for subtotal colectomy (Black and Sholl, 1954; Scarborough, 1955; Goligher, de Dombal, Graham, and Watkinson, 1967) in the surgeon's judgment the patient might not tolerate the additional trauma of a rectal dissection while an elective resection can be done later with much less risk. This is certainly true when the indication for surgery is colonic perforation or toxic dilatation. When there are perirectal suppurative complications it might also be wise to permit the side tracking of the faecal content for a period before excising the rectum.

In most instances, however, an ileostomy and subtotal colectomy is favoured as the procedure of choice without a definitive plan for later management of the distal segment. In some cases it is intended to leave the rectum in situ indefinitely, in the hope that the aetiology of ulcerative colitis will soon be discovered and hence the ileostomy need not be permanent. This is an optimistic and impractical attitude. In others abdominoperineal resection is planned for some months after subtotal colectomy when the general condition of the patient has improved. In still others the primary intention in leaving the rectal segment is to utilize it for a reanastomosis at a later date. Actually an indication for abdominoperineal resection arises in $40 \%$ of patients within one year and in $90 \%$ within three years. In only three instances $(2.0 \%)$ in the present study was a reanastomosis considered feasible. It is of interest that in at least two of these three patients the rectum was initially free of disease, suggesting the possibility of granulomatous rather than ulcerative colitis. Since pathological examination of the colon following subtotal colectomy did not reveal the characteristic features of granulomatous disease, these cases had to be included in the study though with reservations. One of these was the only patient out of the original 136 in whom the rectum has remained free of disease.

There has been no controlled study of the results of treatment of the isolated rectal segment with steroid solution, but in our own experience the response has not been favourable. The number of rectums in which the mucosal appearance returns to normal must be very few.

One controversial issue concerning the rectal segment in ulcerative colitis then is whether it should ever be reanastomosed to the small bowel. In an earlier study by Garlock and Lyons, a reanastomosis failed in three out of the seven cases in which it was attempted (Garlock and Lyons, 1954). This means, however, that it was successful, at least for an extended period, in four out of seven. The experience 
following reanastomosis has been universally poor; in all but a small minority of cases this procedure is followed by recurrent ulcerative proctitis, often fulminating, and a second ileostomy'is required. Since in years past no histological distinction was made between ulcerative and granulomatous colitis, perhaps some or all of the temporary successes were in cases of granulomatous disease. In at least $72 \%$ of those cases of inflammatory disease of the colon initially sparing the rectum, the colonic pathology has been proven to be granulomatous (Korelitz, 1967).

In cases of ulcerative colitis requiring surgical intervention, the fate of the rectal segment should be planned. Except in the instances when a primary ileorectal anastomosis seems desirable, the rectum should be resected during the initial operation if feasible.

\section{SUMMARY}

Of 136 patients with ulcerative colitis treated by ileostomy and subtotal colectomy, $126(94 \%)$ later underwent abdominoperineal resection, $40 \%$ within one year and $90 \%$ within three years. In an addi- tional seven patients the rectal disease persisted. The remaining three had reanastomoses, only one without recurrence thereafter. The mortality rate following secondary abdominoperineal resection was $3 \%$. One patient developed carcinoma in the rectal segment.

Since the rectal segment involved with ulcerative colitis can rarely, if ever, be utilized for a successful reanastomosis following a previous subtotal colectomy, in most cases a primary total proctocolectomy is indicated.

\section{REFERENCES}

Black, B. M., and Sholl, P. R. (1954). Surgical treatment of chronic ulcerative colitis. Arch. Surg., 68, 454-462.

Garlock, J. H., and Lyons, A. S. (1954). The role of surgery in the therapy of ulcerative colitis. Gastroenterology, 26, 709-722.

Goligher, J. C., de Dombal, F. T., Graham, N. G., and Watkinson, G. (1967). Early surgery in the management of severe ulcerative colitis. Brit. med. J., 3, 193-195.

Korelitz, B. I. (1967). Clinical course, late results and pathological nature of inflammatory disease of the colon initially sparing the rectum. Gut, 8, 281-290.

Mayo, C. W., Fly, O. A., Jr, and Connelly, M. E. (1956). Fate of the remaining rectal segment after subtotal colectomy for ulcerative colitis. Ann. Surg., 144, 753-757.

Moss, G. S., and Keddie, N. (1965). Fate of rectal stump in ulcerative colitis. Arch. Surg., 91, 967-970.

Scarborough, R. A. (1955). Surgical treatment of chronic ulcerative colitis. Amer. J. Surg., 89, 1224-1229. 